\title{
DREIMT: a drug repositioning database and prioritization tool for immunomodulation.
}

Kevin Troulé ${ }^{1 \dagger}$, Hugo López-Fernández ${ }^{2,3 \dagger}$, Santiago García-Martín ${ }^{1}$, Miguel Reboiro-Jato ${ }^{2,3}$, Carlos CarreteroPuche ${ }^{1}$, Jordi Martorell-Marugán ${ }^{4}$, Guillermo Martín-Serrano ${ }^{1}$, Pedro Carmona-Sáez ${ }^{4}$, Daniel GonzálezPeña ${ }^{2,3}$, Fátima Al-Shahrour ${ }^{1, *}$ and Gonzalo Gómez-López ${ }^{1, *}$.

${ }^{1}$ Bioinformatics Unit, Spanish National Cancer Research Centre (CNIO), Madrid 28029, Spain.

${ }^{2}$ SING Research Group, CINBIO - Biomedical Research Centre (University of Vigo), IIS Galicia Sur - Galicia Sur Health Research Institute (SERGAS-UVIGO). ${ }^{3}$ Department of Computer Science, University of Vigo, Ourense, Spain

${ }^{4}$ Centre for Genomics and Oncological Research (GENYO), Granada, Spain.

${ }^{\dagger}$ Contributed equally.

*To whom correspondence should be addressed.

\section{Abstract}

Motivation: Drug immunomodulation modifies the response of the immune system and can be therapeutically exploited in pathologies such as cancer and autoimmune diseases.

Results: DREIMT is a new hypothesis-generation web tool which performs drug prioritization analysis for immunomodulation. DREIMT provides significant immunomodulatory drugs targeting up to 70 immune cells subtypes through a curated database that integrates 4,960 drug profiles and $2,6 \mathrm{~K}$ immune gene expression signatures. The tool also suggests potential immunomodulatory drugs targeting usersupplied gene expression signatures. Final output includes drug-signature association scores, FDRs and downloadable plots and results tables.

Availability: http://www.dreimt.org

Contact: falshahrour@cnio.es; ggomez@cnio.es 


\section{Introduction}

Immune system dysregulations have been related to a wide spectrum of complex diseases such as autoimmune disorders and cancer. Autoimmunity processes are clinically diverse and they are distinguished by an immune-mediated attack on the body's own tissues led by self-reactive B and T cells (Rose et al. 2016). In cancer, the intercellular signalling between the malignant cells and certain immune cell subpopulations (e.g. regulatory T cells (Tregs) and tumor-associated macrophages (TAMs)) contributes to tumor microenvironment immunosuppression fostering cell proliferation and tumor evasion (Stockis et al. 2019; Linde et al. 2018). The presence of Tregs and TAMs subpopulations in tumor microenvironment has been also correlated with cancer poor prognosis in contrast to better prognosis shown by those tumors with high rate of tumor-infiltrated CD8+ T cells (Fridman et al. 2017). This has led to propose immune cells targeting as a therapeutic strategy. For instance, some studies have shown that activity of specific immune cell populations can be targeted using systemic or non-systemic approaches (e.g. drugconjugated nanoparticles) thus enhancing the outcome of some tumors and autoimmune diseases (Lu et al. 2020; Riley et al., 2019; Genovese et al., 2016). Recently, drugmediated immunomodulation has been proposed as a therapeutic approach in patients with COVID-19 associated cytokine storm (Richardson $P$ et al., 2020). This highlights the interest in developing new methodologies to propose immunomodulatory therapeutic strategies capable of selectively affect the function of specific immune cells. Here we introduce DREIMT (Drug REpositioning for IMmune Transcriptome), a tool for hypothesis generation of drugs capable of modulating (boosting or inhibiting) the immune cells activity.

\section{Methods and features}


DREIMT integrates $~ 2.6 \mathrm{k}$ immune gene expression signatures with a collection of 4690 consensus drug profiles (Perales-Patón et al., 2019) from the catalog of drug perturbations expression profiles in cancer cell lines from the LINCS L1000 dataset (Subramanian et al., 2017) (Figure 1). Immune signatures were obtained from published studies and public databases covering up to 70 immune cell subtypes (Suppl. Materials S1-3, T1). DREIMT performs a fgsea to test the significance of the immune signature enrichment across the ranked genes of each drug profile (Korotkevich et al., 2020). The fgsea enrichment scores are used to calculate the Drug Prioritization score (т) as described elsewhere (Subramanian et al., 2017), comparing the obtained enrichment score for a particular signature to the rest of immune signatures. The $\mathrm{T}$ score allows the prioritization of drugs to target immune cells. DREIMT also includes a Drug Specificity Score (DSS) that summarises the cell-specificity of a given drug across multiple cancer cell lines (Hodos et al., 2018; Suppl. Materials S4-6). Significant drug-immune signature associations $(|\mathrm{T}|>80)$ are stored in the DREIMT database (DREIMTdb) accessible through a RESTful API. We have validated $>20$ DREIMT hypothesis by scientific literature (Suppl. Materials S7, T2). 
bioRxiv preprint doi: https://doi org/10.1101/2020.06.24.168468; this version posted June 24, 2020. The copyright holder for this preprint (which was not certified by peer review) is the author/funder, who has granted bioRxiv a license to display the preprint in perpetuity. It is made available under aCC-BY-NC 4.0 International license.

Fig. 1. DREIMT workflow.

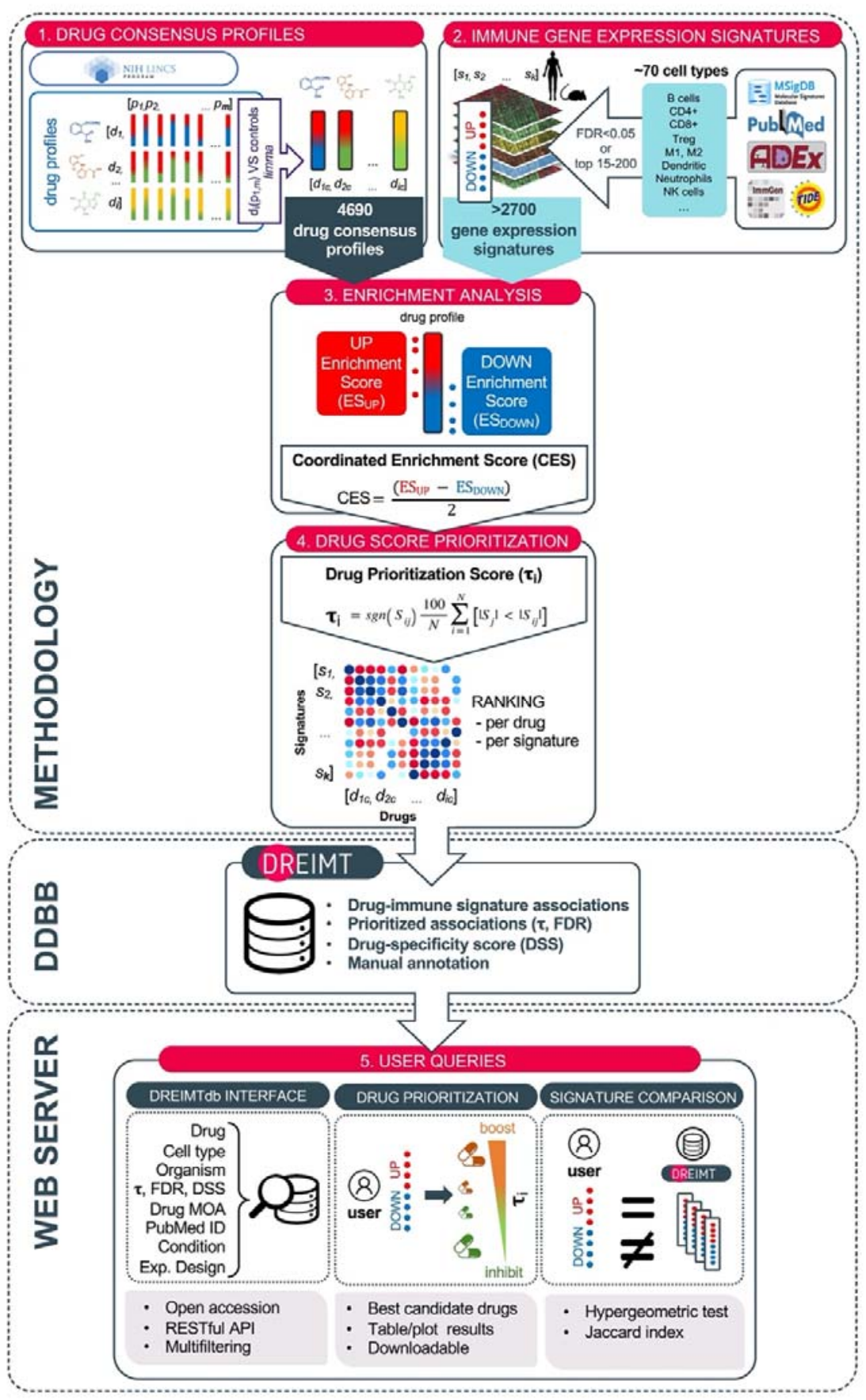




\section{DREIMT web server}

DREIMT web server functionalities include DREIMTdb interface, drug prioritization and signature comparison tools (Figure 1). DREIMTdb interface allows an easy interrogation of the drug-immune signature associations allowing multi-filtering for advanced queries. Drug prioritization functionality allows the search for the best candidate drugs to boost or inhibit the user-defined immune gene expression signature of interest. Drug results are prioritized by $\mathrm{T}$ and DSS and filtered by statistical significance (FDR $<0.05)$. Final outputs are displayed as fully downloadable tables and plots. Additionally, users can compare their own gene signatures against full DREIMTdb contents. As a result, the tool provides the similarity score (jaccard index) and FDR value per each signature compared (Suppl. Materials S8,9). Implementation details and links to the source code repositories, are provided in the Suppl. Materials S10.

\section{Conclusions}

Drugs affect the biology and activity of the immune system, however such interactions are often poorly understood. We have developed DREIMT, a tool to allow users to generate hypotheses and explore novel druggable targets across the immune system, thus supporting drug repositioning leveraging transcriptomics data. DREIMT is fully accessible at http://www.dreimt.org.

\section{Acknowledgements}

José Antonio López for the fruitful discussions for the development of the DREIMT concept. BU staff for beta testing and DREIMTdb curation. 


\section{Funding}

BU is supported by the Instituto de Salud Carlos III (ISCIII); Spanish National Bioinformatics Institute (PT17/0009/0011 - ISCIII-SGEFI / ERDF); RETOS (RTI2018-097596-B-I00); Paradifference Foundation. K.T is supported by Severo Ochoa FPI; S.G-M is supported by Comunidad de Madrid [PEJD-2019-PRE/BMD-15732]. H.L-F. is supported by C. de Educación, Universidades e Formación Profesional (Xunta de Galicia); ED431C2018/55GRC Competitive Reference Group. P.C-S is supported by Junta de Andalucía [PI-01732017].

Conflict of Interest: none declared.

\section{References}

Fang, R. H. \& Zhang, L. (2016) Nanoparticle-Based modulation of the immune system. Annu. Rev. Chem. Biomol. Eng. 7, 305-326.

Fridman WH. et al. (2017) The immune contexture in cancer prognosis and treatment. Nat Rev Clin Oncol. 14(12):717-734.

Genovese MC. et al. (2016) Baricitinib in patients with refractory rheumatoid arthritis. N Engl J Med. 374(13):1243-52.

Hodos H. et al. (2018) Cell-specific prediction and application of drug-induced gene expression profiles. Pac Symp Biocomput. 23: 32-43.

Korotkevich G, et al. (2019) Fast gene set enrichment analysis. bioRxiv 060012; doi: https://doi.org/10.1101/060012

Linde N. et al. (2018). Macrophages orchestrate breast cancer early dissemination and metastasis. Nat Commun. 9(1):21. 
Lu Y. et al. (2020) Complement signals determine opposite effects of B cells in chemotherapy-induced immunity. Cell. 180(6):1081-1097.

Perales-Patón, J., et al. (2019). vulcanSpot: a tool to prioritize therapeutic vulnerabilities in cancer. Bioinformatics. 35(22): 4846-4848.

Richardson P. et al. (2020) Baricitinib as potential treatment for 2019-nCoV acute respiratory disease. Lancet. 395: e30-e31

Riley RS. et al. (2019) Delivery technologies for cancer immunotherapy. Nat. Rev. Drug Discov. 18, 175-196.

Rose NR. (2016) Prediction and prevention of autoimmune disease in the 21st Century: A review and preview. Am J Epidemiol. 183(5):403-6.

Stockis J. et al. (2019) Regulation of regulatory T cells in cancer. Immunology, 157, 219231.

Subramanian A. et al. (2017) A next generation connectivity map: L1000 platform and the first 1,000,000 profiles. Cell, 171, 1437-1452. 




